\title{
EXPERIMENTAL ANALYSIS OF THE SHOCK DYNAMICS ON A TRANSONIC LAMINAR AIRFOIL
}

\author{
V. Brion, J. Dandois, J.-C. Abart, and P. Paillart \\ ONERA, The French Aerospace Lab. \\ Centre de Mendon \\ 8 rue des Vertugadins, Mendon 92190, France
}

\begin{abstract}
This paper describes an experimental analysis of the buffet phenomenon on a two-dimensional (2D), transonic, and laminar airfoil at a Reynolds number around $3 \cdot 10^{6}$. Investigations are carried out in ONERA's S3Ch transonic wind tunnel. The experimental setup allows to vary the Mach number, the angle of attack, and the state of the boundary layer upstream of the shock which can be turbulent or laminar depending on the presence of artificial tripping. Buffet occurs when either the angle of attack or the Mach number is set above a given threshold, which depends upon the particular airfoil, and, as shown here, on the state of the boundary layer. Above the threshold, the boundary layer / shock interaction destabilizes, causing the oscillation of the entire flow field. In the turbulent case, the shock wave moves back and forth over a significant portion of the chord at a frequency of about $75 \mathrm{~Hz}$ corresponding to a chord based on Strouhal number $S_{t} \simeq 0.07$, in agreement with previous researches on this phenomenon. In the laminar case, a similar unsteady situation occurs but at a frequency much higher, about $1130 \mathrm{~Hz}$, which corresponds to a Strouhal number of about $S_{t} \simeq 1$. Flow oscillations are limited to the shock foot, the shock itself moving only lightly. The turbulent and laminar buffet thresholds are provided. An attempt to apply the classical feedback loop scenario to explain the unsteadiness of the flow in the laminar case is carried out but shows a deceptive agreement with the experimental data. Two other mechanisms of unsteadiness are additionally explored, one based on vortex shedding behind the airfoil and the other on the possible breathing of the laminar separation bubble, which give valuable insights into the flow physics.
\end{abstract}

\section{INTRODUCTION}

In transonic flow past airfoils, shock wave / turbulent boundary layer interactions may lead to powerful oscillations of the entire flow field when exceeding the critical Mach number or the critical angle of attack $\left(M_{c}, \alpha_{c}\right)$. This phenomenon called buffet is characterized by large shock displacements and periodic flow separation between the shock foot and the trailing edge. While turbulent buffet has received

(C) The authors, published by EDP Sciences. This is an Open Access article distributed under the terms of the Creative Commons Attribution License 4.0 (http://creativecommons.org/licenses/by/4.0/). 
much attention in the past (see, for example, [1-4]), there is little knowledge on the dynamics of the laminar boundary layer / shock interaction. This question is of fundamental importance to the use of laminar flows for drag reduction, one of the key target to limit the environmental footprint of aviation. Previous works on this topic (see, for instance, [5]) suggest that laminar flows do not exhibit the shock oscillations characteristic of the turbulent case but further analysis is still needed to fully clarify the laminar dynamics and discuss these findings.

One of the goal of the present experiment is to document an experimental database for transonic flows over laminar airfoils including the buffet phenomenon. Except direct numerical simulation (DNS) (but it is not affordable yet for such high Reynolds number flows), all numerical methods rely on theoretical models complemented with empirical data to capture boundary layer transition. The development of well calibrated data to validate these models is a requirement to bring these tools to an industrial level and allow the design of aircraft with larger zones of laminar flow. Capturing unsteady transition is another issue which does not seem to have ever been addressed. Current simulation tools are able to capture turbulent buffet (see, for instance, [6-8]) but it is currently unknown whether they are successful to capture the flow dynamics when the boundary layer is only partially turbulent. The second objective of the work is to question the very existence of laminar buffet, i. e., the existence of a phenomenon consisting of global and low frequency flow fluctuations in a given region of $\left(M_{0}, \alpha\right)$ space. In the present paper, the experimental evidence of a laminar phenomenon quite analogous to turbulent buffet is provided. However, the laminar phenomenon features a frequency an order of magnitude higher than the turbulent buffet and little shock displacement, which questions its physics.

The turbulent buffet causes large and periodic movements of the shock, combined with separation of the flow between the shock and the trailing edge. The attempts to understand its physics have faced the difficulty of describing a global dynamics. One of the earliest explanation relies on a resonance between the hydrodynamic instability waves emitted behind the shock wave [1-3] and the feedback provided by their impact on the trailing edge, which generates upstream traveling acoustic waves. These waves amplify the instability when they hit the shock, creating the loop of amplification which then saturates through the nonlinear effects. More recent work associates the buffet phenomenon with a global unstable mode of the flow [4]. In the future, similar efforts will have to be undertaken in the transition free (laminar) scenario to provide such a modal point of view. In the present work, a phenomenological analysis including an attempt to apply the acoustic feedback scenario, will be carried out to describe the experimental observations.

The experimental arrangement is described in the first section of the paper. The second section is devoted to the airfoil aerodynamics, which is described on the basis of steady pressure measurements for various conditions of angle of 
attack and Mach number. The third section describes the turbulent buffet phenomenon when the transition is forced and then presents the dynamics observed in the free transition setup. High-speed Schlieren movies and unsteady pressure signals at the wing surface are used for this analysis. The fourth section is a discussion on the possible physical mechanisms, including the acoustic feedback loop scenario, to explain laminar buffet.

\section{EXPERIMENT}

\subsection{Setup}

The experiments are carried out in ONERA's S3Ch wind tunnel. This facility operates at Mach numbers in the range $[0.1,1.2]$ with a two stages fan powered by a 3.5-megawatt engine. The mechanical power transmitted to the flow is adjusted by pitching the fan blades while the rotating speed is held constant. The fluctuations in Mach number are minimized by the use of a sonic throat placed downstream of the test section and activated above $\mathrm{M} \simeq 0.6$ allowing $\Delta \mathrm{M}=10^{-3}$ in this case. The tunnel is operated at atmospheric stagnation pressure $P_{0} \simeq P_{a}$. The stagnation temperature $T_{0}$ is constant at about $310 \mathrm{~K}$ thanks to a heat exchanger placed upstream of the settling chamber which discharges the heat accumulated inside the tunnel by wall friction and engine heat-up. The test section is rectangular with a height of $0.763 \mathrm{~m}$, a width of $0.804 \mathrm{~m}$, and a length of $2.2 \mathrm{~m}$. It is equipped with adaptive upper and lower walls in order to create infinite flow field conditions, thus minimizing wall interferences and providing constant Mach number and flow incidence at the location of the model. The coordinate system that is used is as follows : $(O x)$ points downstream in the direction of the free stream; $(O y)$ is horizontal to the right when one faces the flow; and $(O z)$ is vertical pointing upward.

The flow Reynolds number is $\operatorname{Re}=\rho_{0} U_{0} c / \mu_{0} \simeq 3 \cdot 10^{6}$ where $\rho_{0}$ and $\mu_{0}$ are the local mass density and viscosity, the latter being obtained by the Sutherland's law as a function of local temperature $T_{0}$. The Mach number is $\mathrm{M}_{0}=U_{0} / c_{0}$ where $c_{0}=\left(\gamma R T_{0}\right)^{1 / 2}$ is the local speed of sound. The dynamic pressure of the incoming flow is $q_{0}=0.5 \rho U_{0}^{2}$. The Mach number $\mathrm{M}_{0}$ and the angle of attack $\alpha$ are the main parameters of the flow. The angle of attack has been varied in the range $\left[0.5^{\circ} ; 4^{\circ}\right]$. At each angle of attack, the Mach number has been varied in the range $[0.69 ; 0.8]$ with small increments. In addition, the state of the boundary layer developing on the upper surface has also been varied, while the lower surface was imposed turbulent by artificial tripping at $7 \%$ of chord. On the upper surface, the boundary layer has been either kept naturally developing, or artificially tripped at $7 \%$ and $40 \%$ of chord, thus providing three different boundary layer configurations. The tripping device is tape with a saw tooth shape. The thickness of the tape is $e_{t 7}=0.1 \mathrm{~mm}$ when installed at $7 \%$ and $e_{t 40}=0.205 \mathrm{~mm}$ when installed at $40 \%$. 


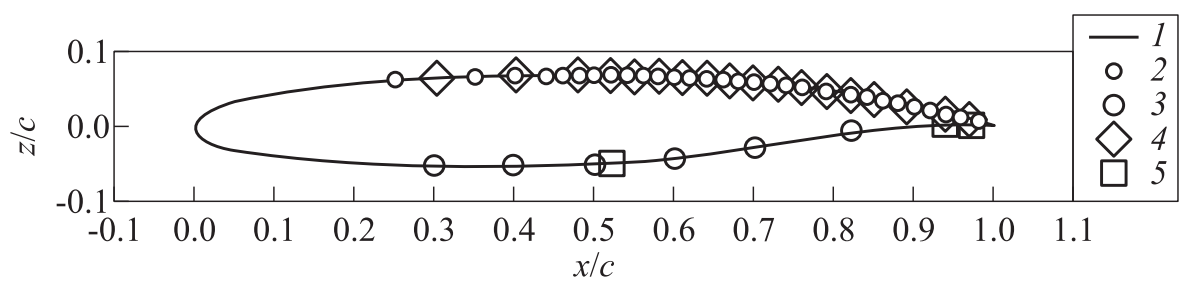

(a)

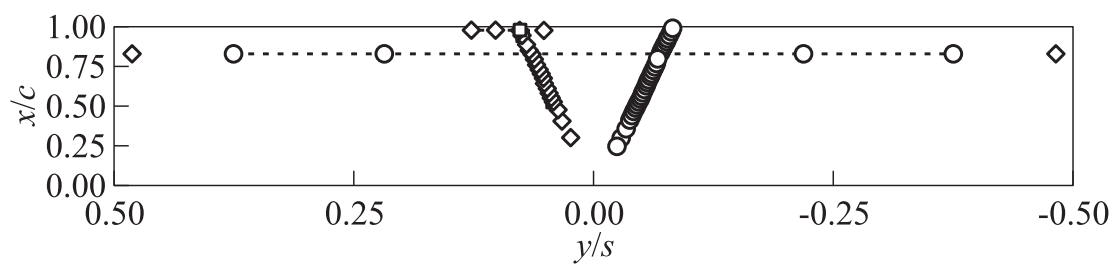

(b)

Figure 1 Geometry of the airfoil including the equipment of pressure taps (1 upper surface and 2 - lower surface) and transducers (3 - upper surface and 4 lower surface) at location $\boldsymbol{x}_{t, i=1,41}$ and $\boldsymbol{x}_{k, i=1,26}$ (solid curve - OALT25): (a) side view; and (b) top view;

Figure 1 shows the airfoil used for the test, an ONERA designed airfoil named OALT25. It is a laminar supercritical airfoil with a relative thickness of $12.18 \%$ and a thick trailing edge of $0.5 \%$ of chord. It is a full span model with a chord $c=230 \mathrm{~mm}$. The wing is attached to the wind tunnel outside the test section, leaving the test chamber clean of any support. The angle of attack is adjusted in two steps: first, the external support of the model is rotated (increments of $0.5^{\circ}$ are allowed at this stage) and then, precise adjustment in the range $\pm 0.5^{\circ}$ is achieved by tilting the adaptive walls.

\subsection{Measurements}

The pressure equipment is shown in Fig. 1. There are 41 pressure taps and 26 pressure transducers. Pressure taps are located at the points $\boldsymbol{x}_{t, i}=\left(x_{t, i}, y_{t, i}, z_{t, i}\right)$ for $i=1, \ldots, 41$ corresponding to a chordwise and spanwise distributions. Their locations have been chosen so as to capture the wing aerodynamics and the flow two-dimensionality, respectively. Pressure transducers are located at the positions $\boldsymbol{x}_{k, i}=\left(x_{k, j}, y_{k, j}, z_{k, j}\right)$ for $j=1, \ldots, 26$ corresponding to a mostly chordwise distribution. Several of them are packed at the trailing edge in order to monitor flow separation there, which is a precursor of buffet [3]. Differential pressure transducers of range 1 PSI (pound per square inch) are used at the trailing edge and absolute pressure transducers of range 15 PSI everywhere else. 
Pressure signals from the transducers are sampled at $f_{s}=10 \mathrm{kHz}$ during $T_{s}$ $=40 \mathrm{~s}$ and low-pass filtered at $4000 \mathrm{~Hz}$ prior to digital conversion. Pressure signals from the taps are obtained from an electronic pressure scanner and averaged over $5 \mathrm{~s}$. The two transducers at the edge of the model in the spanwise direction help to monitor possible unsteadiness in the corner flow with the side walls (none was detected). It should be noted that the chordwise distributions are inclined with respect to the incoming flow to prevent cross contamination between the holes and to minimize the perturbations to the boundary layer. In addition, the first $20 \%$ of chord are free of any roughness.

A picture of the experimental apparatus is laid out in Fig. 2. It shows the mounting of the wing in between the side walls of the test section. The wing has been specifically designed for these tests. The side windows, suited for Schlieren imaging, are wide discs that allow a view on much of the flow above the wing and downstream. High-speed recording of the Schlieren stream of data has been achieved for each test at a rate of 6000 frame/s.

An infrared (IR) camera is installed outside the test section, with a view on the model through the IR window and by reflection on the roof of the tunnel, as indicated in Fig. 2. This original setup provides a view on approximately a quarter of span starting from mid-span. It has been used because the adaptive walls prevented a direct view from above. The IR camera is the SC5000 model from FLIR. Its sensor resolution is $640 \times 512$ pixels and $20 \mathrm{mK}$ in temperature. It operates in the short infrared range

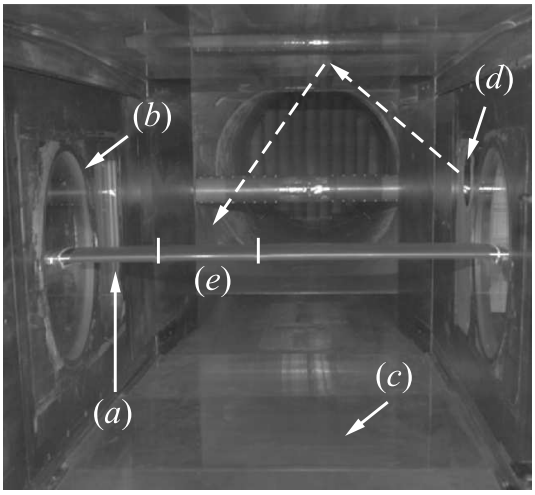

Figure 2 Photograph of the wing inside the test section taken from upstream: (a) - wing covered with black paint to increase emissivity for IR imaging; (b) Schlieren window; $(c)$ - adaptive lower wall; $(d)$ - IR window; and $(e)$ - optical path for the IR imaging and spanwise area which is monitored

from 2.5 to $5.1 \mu \mathrm{m}$. The camera is run at $100 \mathrm{~Hz}$ with an exposure time of about $1 \mu \mathrm{s}$. A set of 100 images is recorded at each acquisition.

Thermographic cameras detect radiations and deliver the associated power flux integrated over the pixels of the sensor. In the current setup, the model has been painted in black to increase emissivity to a value close to unity. The Stefan-Boltzmann law allows to retrieve the temperature from the measured flux. Here, the calibration law for a black body given by the manufacturer of the camera has been used to do this conversion.

A preliminary analysis of the sources of heat transfer between the fluid and the solid indicates that the wall temperature results from numerous effects that 


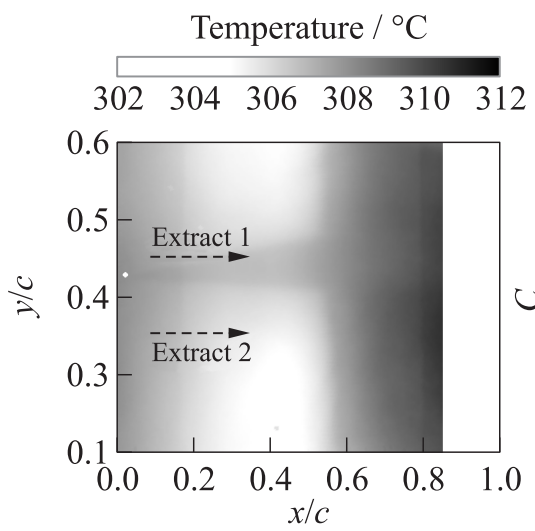

(a)

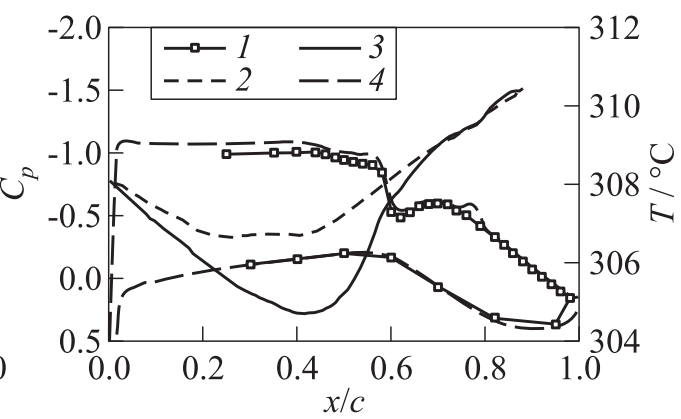

(b)

Figure 3 Analysis of the mean IR image obtained for $\left(\mathrm{M}_{0}, \alpha\right)=\left(0.745,1.5^{\circ}\right)$ with an isolated roughness: (a) mapped IR image; the roughness is indicated by the white dot; the field is blanked after $85 \%$ of chord because the trailing edge area was not painted; and $(b)$ combined mean pressure coefficient (1) and temperature evolutions along the chord $(2$ - extract 1 , downstream of the added roughness; 3 - extract 2 , outside the added roughness wake; and 4 - CFD RANS AHD criterion)

are difficult to separate without any dedicated protocol. First, there is the diffusion of heat inside the solid. It depends on the particular design of the wing. In the present setting, the wing is not plain as it is composed of several pieces and also features a few empty spaces to allow for the equipment which makes the diffusion process nontrivial. Temperature also depends upon the diffusion inside the fluid and upon convective transfer and heat production by viscous friction inside the boundary layer. It should be also mentioned that the potential flow around the wing produces a nonuniform temperature field. In particular, the presence of a shock leads to a sudden increase of temperature. A reasonable approximation over all this, which is validated by the IR images (Fig. 3), is to assume that the situation is mostly $2 \mathrm{D}$, owing to the large aspect ratio of the wing. In particular and except at the wing tips, heat transfer with the tunnel walls can be neglected.

It is not the point of the present paper to distinguish between all these sources in the temperature field measured by the camera. Instead, thermography has been used to check $(i)$ that the flow was free from any spurious transition and, in particular, $(i i)$ that the forced free cases were laminar up to the shock. For such an analysis, the raw IR images are sufficient as is shown later on.

The postprocessing procedures of the IR raw images is the following. First, an average over the set of 100 images is calculated. Then, a mapping of the mean to the airfoil coordinate system is achieved. The initial mapping process 
has been set with an image of the model in the absence of flow (wind tunnel off) that disposed of 6 markers points distributed on the visible part of the upper surface (see label (e) in Fig. 2). Then, the parameters of the mapping process were adapted to each $\left(\mathrm{M}_{0}, \alpha\right)$ point because of the variations in the shape of the upper wall and because of the deformation of the wing induced by the aerodynamic loads. The first step is managed automatically and the second one manually.

The quality of the thermographic monitoring has been first established on an artificially tripped configuration, using an isolated roughness placed at about $5 \%$ of chord as a target. The IR image, temperature, and pressure profiles are shown in Fig. 3 for the case $\left(\mathrm{M}_{0}, \alpha\right)=\left(0.745,1.5^{\circ}\right)$. The pressure coefficient $C_{p}$ is defined by

$$
C_{p}=\frac{p-p_{0}}{q_{0}}
$$

The $C_{p}$ distribution shows that the flow exhibits a shock wave at $60 \%$ of chord. One can also observe a slight compression ahead of the shock and an acceleration downstream of it.

A transition cone forms as a result of the isolated roughness (which is marked by a white dot in the image, see Fig. $3 a$ ) - the pressure measurement is in the laminar region, not in the cone. The cone angle is approximately $10^{\circ}$. Two chordwise profiles of temperature are provided, the first one in the region of the cone and the other outside. It can be noted that the temperature initially decreases downstream of the leading edge. The leading order effect on this decrease is the acceleration of the flow which leads to a drop in local temperature. About mid-chord, the effect reverses, as the flow decelerates upstream of the shock, and wall temperature increases. The detail of this evolution is different in the cone region and outside. In the cone, temperature decreases less as a consequence of the production of heat brought about by the increased friction of the turbulent motion. It also exhibits a plateau region about the location of the slope reversal. Downstream of the shock, both temperature profiles merge to a single curve, indicating that powerful phenomena at the shock foot cause important heat transfers that level out upstream differences.

\subsection{Laminar Separation Bubble}

As noted earlier, the $C_{p}$ distribution is marked by a slight compression ahead of the shock, at about $40 \%$ of chord. This shape in the $C_{p}$ distribution only appears in the pressure measurements performed in transition free (laminar) cases. The most likely reason for this is the existence of a laminar separation bubble under the shock foot, which forms as soon as the compression occurs as a result of the little resilience of a laminar boundary layer to an adverse pressure gradient. An annotated $C_{p}$ distribution is provided in Fig. $5 d$ later in the text, 
which shows the location of this compression, that of the flow separation, and the approximate extent of the bubble. The characteristics of the bubble are evaluated with the help of numerical Reynolds-averaged Navier-Stokes (RANS) results described below because experimental data do not allow to finely qualify them. In particular, it is considered that laminar separation occurs about $5 \%$ downstream of the first compression before the shock and reattaches at the same location as the shock.

Schlichting and Gersten [9] explain that in a laminar boundary layer, the compression imposed by the shock diffuses upstream over a distance called the interaction length. This length is roughly the distance from the shock location to the laminar separation. Boundary layer separation initiates the formation of a bubble which reattaches downstream thanks to the increased diffusion brought about by the turbulence which naturally develops in the shear layer at the top of the bubble.

The existence of a laminar separation bubble is confirmed by Schlieren images (see, for instance, Fig. 7) where it corresponds to the dead air region below the shock foot and by numerical simulations. In particular, complementary RANS calculations were performed that confirm this scenario. These simulations were performed with the elsA software developed by ONERA and are based on the Spalart-Allmaras one-equation model [10]. Natural transition is computed

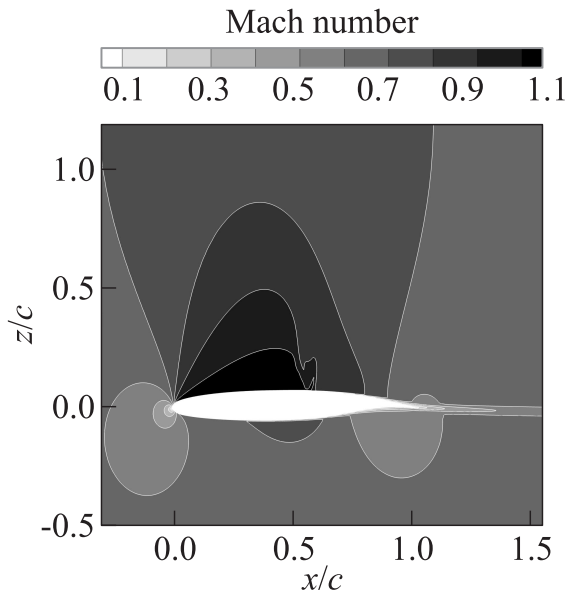

(a)

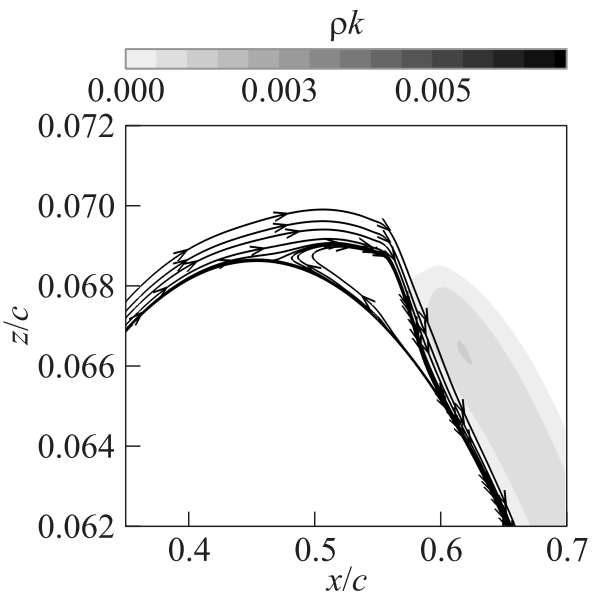

(b)

Figure 4 The RANS calculated flow field about the OALT25 airfoil for the experimental case at $\left(\mathrm{M}_{0}, \alpha\right)=(0.745,1.5)$ : $(a)$ contours of Mach number; and $(b)$ zoom in the laminar separation bubble region with an indication of the flow streamlines and contours of $\rho k$, the turbulent kinetic energy. Note that the horizontal and vertical scales are independent in frame $(b)$ to highlight the bubble 
with a supersonic extension of a combination of the Arnal-Habiballah-Delcourt (AHD) [11] and Gleyzes et al. [12] criteria. The CFD results are shown in

Fig. 4. The transition criterion performs well in this steady case. As shown in Fig. $3 b$, the pressure distribution satisfactorily matches the experiment, although the pressure level in the plateau region upstream of the shock is slightly lower, and the compression prior to the shock occurs a little more downstream. The contours of Mach number of the flow past the wing are shown in Fig. $4 a$ and a zoom on the shock foot is provided in Fig. $4 b$. The latter exhibits the laminar separation bubble and the developing turbulence at its downstream side.

It is interesting from these simulations to evaluate the characteristics, size, and location of the laminar separation bubble. The flow streamlines indicate that laminar separation occurs at $46 \%$ of chord, which is approximately $5 \%$ of chord downstream of the location of the increase in pressure in the $C_{p}$ distribution. Flow reattachment occurs at the shock position, i.e., $60 \%$ of chord, making a bubble length of about $l=0.15 c$. The bubble maximum thickness is about $h=0.002 c$, which is about $0.5 \mathrm{~mm}$ in physical units, yielding and aspect ratio of about 75 .

\section{RESULTS}

\subsection{Mean Surface Pressure}

Figure 5 shows the pressure distribution on the wing for a selection of angles of attack and $\mathrm{M}_{0}=0.73$. The transition free (laminar) and forced at $7 \%$ (turbulent) cases are considered. Figure $5 d$ is an annotated version of a typical laminar configuration. At low angle of attack (here, $1.5^{\circ}$ ), the flow exhibits only a weak compression wave but not shock. A shock forms when the angle of attack is increased and settles at a position of about $60 \%$ of chord in the transition free (laminar) case and $50 \%$ in the forced transition case. The gap in shock position induced by the boundary layer state is best seen in Fig. $5 c$ which compares the laminar and the turbulent cases for $\alpha=4^{\circ}$. For identical free stream parameters, the shock is significantly more downstream in the laminar case.

Another remarkable point is the extension of the shock in the pressure distribution at $\alpha=4^{\circ}$. It is spread over almost $10 \%$ of chord in the laminar case and $8 \%$ in the turbulent case compared to less than $5 \%$ at the lower angles of attack. From previous analysis of the buffet phenomenon [3], this is suggestive of buffet onset, all the more so as this is correlated with flow separation at the trailing edge, as marked by the divergence of the pressure signal when $\alpha>3.5^{\circ}$ in both laminar and turbulent cases.

Thus, from the flow case $\left(\mathrm{M}_{0}, \alpha\right)=\left(0.73,4^{\circ}\right)$, one expects that increasing either the angle of attack or the Mach will trigger buffet. The shock dynamics 


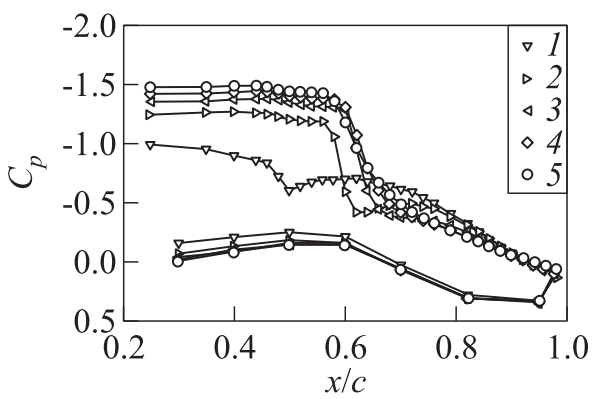

(a)

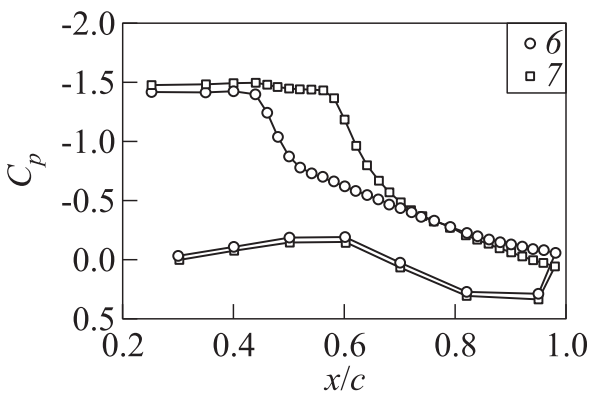

$(c)$

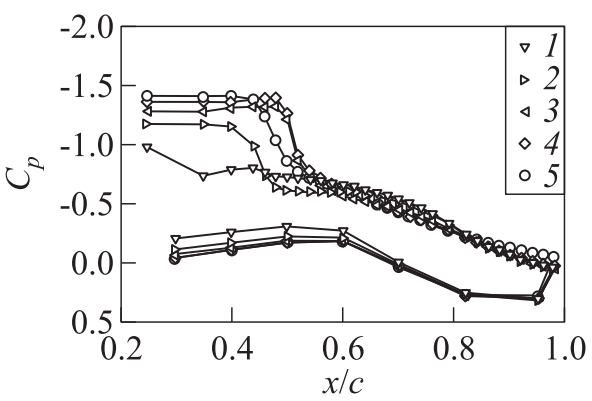

(b)

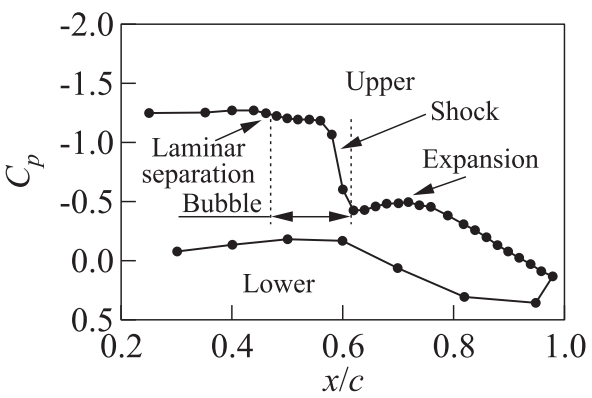

(d)

Figure 5 Evolution of the mean pressure coefficient as a function of chord for $\mathrm{M}_{0}$ $=0.73$ and various angles of attack: $(a)$ transition free (laminar) and $(b)$ transition forced at $7 \%$ (turbulent) of chord $\left(1-\alpha=1.5^{\circ} ; 2-2.5^{\circ} ; 3-3^{\circ} ; 4-3.5^{\circ}\right.$; and $\left.5-\alpha=4^{\circ}\right) ;(c)$ comparison between the laminar and the turbulent case for $\alpha=4^{\circ}$ : forced transition at $7 \%(6)$ and at $0 \%(7)$; and $(d)$ annotated $C_{p}$ distribution in the laminar case, $\alpha=2.5^{\circ}$

for $\left(0.735,4^{\circ}\right)$ is thus analyzed. Figure 6 shows the dynamics of the case with forced transition at $7 \%$ of chord through a sequence of Schlieren images. The frame of these images lies between $x / c \simeq 20 \%$ and $\simeq 110 \%$.

Grid ticks in the figure help to follow the movement of the shock. In frame 1 the shock is initially at its most upstream position. From frame 1 to frame 4 , the shock moves downstream over more than a grid cell. In frame 4 , the shock is at its most downstream location and most intense. In frames 4 and 5 , the flow starts to separate behind the shock owing to the severe compression imposed by the shock. This acts as a lowering of the angle of attack which reduces the effective lift and pushes the shock upstream. As a result, from frame 5 to frame 8 , the shock moves upstream and flow separation becomes massive. In frames 7 and 8 , the shock is back at its most upstream position. It has lost its intensity and thus, the flow starts to reattach behind it. The next step is frame 1 and all 


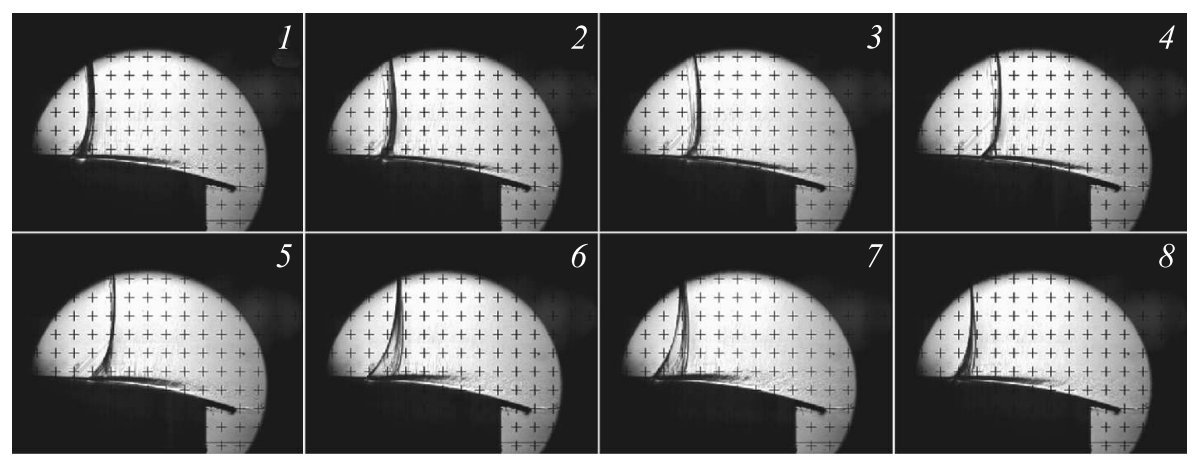

Figure 6 Time sequence over approximately $15 \mathrm{~ms}$ showing the shock dynamics in the turbulent case (tripping $=7 \%),\left(\mathrm{M}_{0}, \alpha\right)=\left(0.735,4^{\circ}\right)$. The grid size is about $8 \%$ of chord

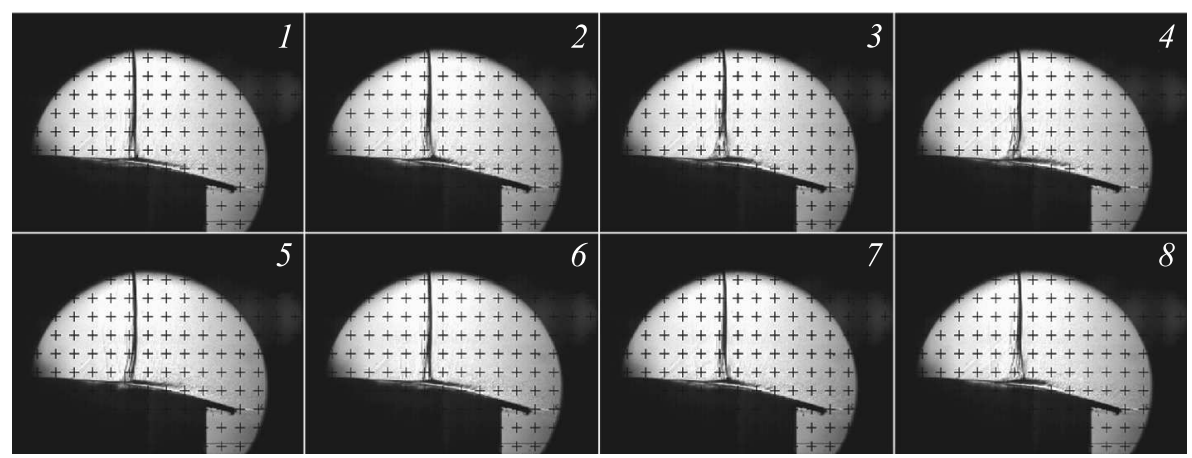

Figure 7 Time sequence over approximately $1.5 \mathrm{~ms}$ showing the shock dynamics in the laminar case (no tripping), $\left(\mathrm{M}_{0}, \alpha\right)=\left(0.735,4^{\circ}\right)$. The grid size is about $8 \%$ of chord

goes periodic in time at a frequency which is close to $70 \mathrm{~Hz}$. This dynamics is essentially $2 \mathrm{D}$ although slight transverse variations are observed, especially when the flow is massively separated (for instance, in frames 6 and 7) as separated flows are intrinsically three-dimensional (3D).

Figure 7 shows the time sequence in the laminar case. Unlike the turbulent case, the shock exhibits only little motion. Most of the unsteadiness is observed at the shock foot. The motion is periodic at a frequency an order of magnitude larger than the turbulent case (the period is about $1.5 \mathrm{~ms}$ vs. $15 \mathrm{~ms}$ in the turbulent case). Flow separation also occurs in relation with the motion of the shock foot. In particular, the development of the flow separation over the entire 


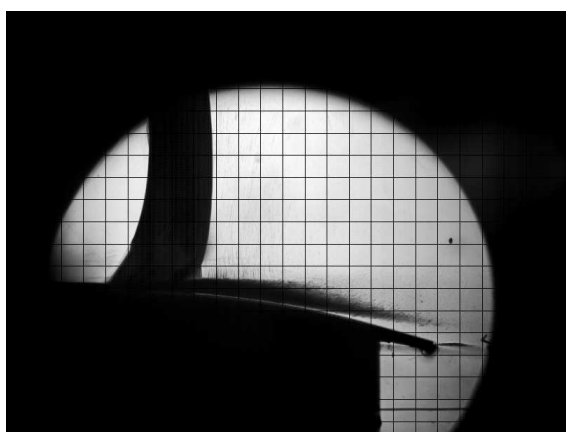

(a)

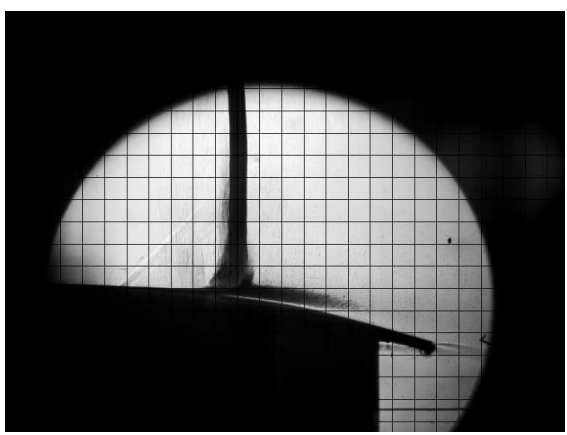

(b)

Figure 8 Minimum intensity projection of the Schlieren movies for the turbulent $(a)$ and laminar $(b)$ cases. The extent of the variations of the shock and of the wake is seen

sequence is similar to the previous description except that the motion to be considered is that of the shock foot. In addition, as noted earlier, the laminar separation bubble can be seen under the shock foot and 3D effects are also present when the flow completely detaches behind the shock.

Figure 8 gives a comparative summary of the laminar and turbulent situations. Every pixel in these images takes the minimum intensity over the entire set of images that compose the Schlieren movies. This allows one to highlight the global footprint of the shock and of the separated flow in two situations. What is remarkable is the extent of the shock in the turbulent case which represents about $24 \%$ of chord, while in the laminar case, the shock moves only about $5 \%$ of chord and the foot over $15 \%$. The vertical extent of the turbulent zone behind the shock is the mark of the periodic separated flow.

\subsection{Spectral Analysis}

A quantitative analysis of the dynamics is carried out by Fourier transforming the unsteady pressure signal. The previous buffet case $\left(\mathrm{M}_{0}, \alpha\right)=\left(0.735,4^{\circ}\right)$ is considered. Figure 9 displays the averaged periodogram of the pressure fluctuations using Hann windowing over subsections of the data and $50 \%$ overlap. Each periodogram is defined as follows:

$$
\hat{P}(f, x)=\frac{f_{s}}{N_{s}}\left|\sum_{n=0}^{N_{s}-1} p(n \Delta t, x) e^{-2 i \pi n f / f_{s}}\right|^{2} .
$$

The number of samples per block used to calculate the periodograms is $N_{s}$ $=4096$, which leads to an averaging over approximately 200 subsections of 


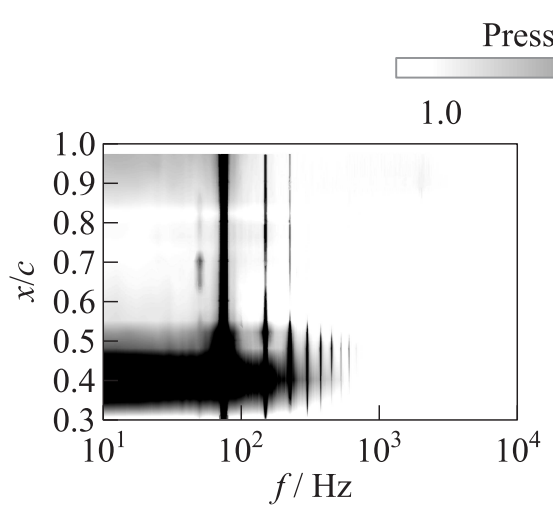

(a)

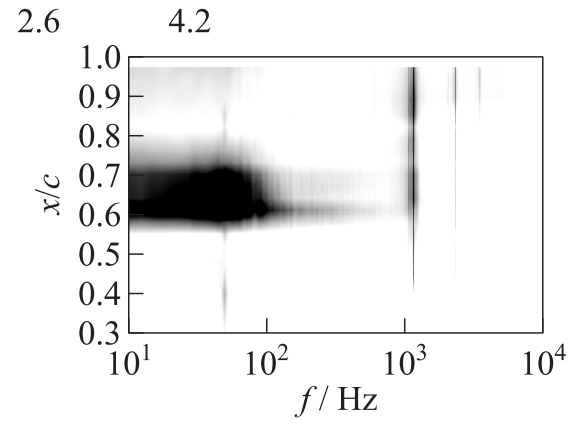

(b)

Figure 9 Contour plot of the spectral component of the pressure fluctuations on the upper surface of the wing. The data are spatially interpolated from the discretely distributed pressure transducers. The spectral component is normalized by $q_{0}$ as $\left\langle\hat{P}^{1 / 2}(f, x)\right\rangle /\left(N_{s} q_{0}\right)$. Turbulent $(a)$ and laminar $(b)$ cases are shown for $\left(\mathrm{M}_{0}, \alpha\right)$ $=\left(0.735,4^{\circ}\right)$. Apart the voltage disturbance at $50 \mathrm{~Hz}$, the peaks that appear in $(a)$ are $f=75,150,225 \mathrm{~Hz}, \ldots$ and in $(b) f=1130,2260$, and $3400 \mathrm{~Hz}$

data. The layout in Fig. 9 details the pressure spectra as a function of chordwise coordinate. These were obtained by interpolation the spectra available at the discrete positions $\boldsymbol{x}_{k, i}$ of the pressure transducers (see Fig. 1). The laminar and turbulent, forced transition at $7 \%$ of chord are considered. The range of $\hat{P}(f, x)$ has been adjusted at best to highlight the discrete peaks of the dynamics. In Figs $9 a$ and $9 b$, the first peak to the left is the 50-hertz voltage disturbance.

In the turbulent case, the entire flow exhibits strong fluctuations at $75 \mathrm{~Hz}$ that correspond to the shock oscillations observed in Fig. 6, i. e., turbulent buffet. The associated chord-based Strouhal number is $S_{t}=0.07$. Many harmonics of this primary frequency appear at the right of the spectrum that feature an amplitude at least an order of magnitude lower. This indicates that the periodicity of the oscillations is very stable in time. It is also remarkable that the frequency matches closely the one found by Jacquin et al. [3] on a different airfoil (OAT15) in the same wind tunnel.

Looking at Fig. $9 b$ and as expected from the previous analysis, the laminar case does not exhibit the low frequency $75 \mathrm{~Hz}$ dynamics. Instead, a primary peak is observed at $1130 \mathrm{~Hz}$ and then repeated with significantly lower amplitudes at 2260 and $3300 \mathrm{~Hz}$. In this case, the chord-based Strouhal number is $S_{t}$ $=1$. This corresponds to the dynamics of the shock foot whose periodic motion appears quite stable too. 
In the two spectra, the primary peak appears over most of the chord, meaning that the dynamics is global. Eventually, two additional features appear in the low-frequency range $(f<100 \mathrm{~Hz})$. The first one is the important level of unsteadiness in the trailing edge region, which, from previous analysis, is to be related to flow separation. The second one is the strong level of unsteadiness around $40 \%$ of chord in the turbulent case and between $60 \%$ and $70 \%$ in the laminar case. This location is slightly upstream of the shock in the turbulent case and almost exactly where the shock occurs in the laminar case. The origin of these fluctuations is unclear at the moment. Part of it could be related to 3D effects which are seen to occur as the shock moves upstream and flow detaches behind.

\subsection{Evolution Towards Buffet}

The evolution of the flow dynamics towards buffet is analyzed by inspecting pressure spectra at increasing angles of attack for a given Mach number; here, $\mathrm{M}_{0}=0.735$. The evolution of the pressure spectra obtained from the transducer located at $x / c=85 \%$ in the turbulent and laminar cases are shown in Fig. 10 . Spectra are normalized by the dynamic pressure $q_{0}$. As expected, at high angle of attack, turbulent cases exhibit buffet at $75 \mathrm{~Hz}$. The laminar flow also fluctuates at about $1130 \mathrm{~Hz}$. Flow oscillation appears earlier in the laminar case; here, at $\alpha=3.5^{\circ}$, all the more so as the Mach is slightly lower in this case $\left(\mathrm{M}_{0}=0.733\right.$, the case $\mathrm{M}_{0}=0.735$ being not available). No distinguishable dynamics occurs below buffet, except a peak at $2000 \mathrm{~Hz}$ in both spectra whose origin is unclear at the moment.

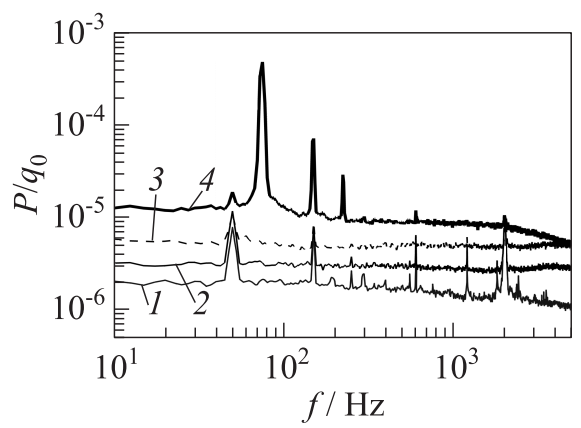

(a)

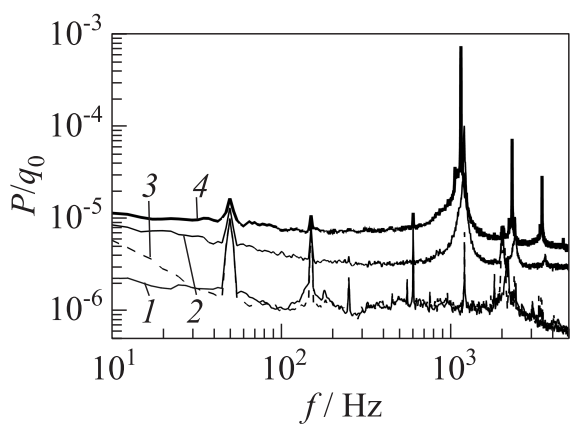

(b)

Figure 10 Spectra of the pressure fluctuations at $x / c=0.85$ for a selection of $\alpha$ $\left(1-1.5^{\circ} ; 2-3^{\circ} ; 3-3.5^{\circ} ;\right.$ and $\left.4-4^{\circ}\right)$ at $\mathrm{M}_{0}=0.735:(a)$ turbulent case; and (b) laminar case 


\subsection{Buffet Threshold}

Previous analysis allows one to clarify the curve $\mathrm{M}_{c}=f_{\mathrm{BL} \text { state }}(\alpha)$ above which buffet occurs. The curve is parametrized by the boundary layer state (turbulent/laminar). Here, only the cases of transition free (laminar) and transition forced at $7 \%$ (turbulent) of chord are considered.

The threshold curve has been determined by considering that the periodic unsteadiness related to buffet is established once a pattern similar to the one exhibited in the case $\left(\mathrm{M}_{0}, \alpha\right)=\left(0.733,3.5^{\circ}\right)$, shown in Fig. 10, appears in the pressure spectra. There is no theoretical background for doing so, and the result partly relies on this arbitrary choice. However, the variations impeded by this method are believed to be small and, therefore, the present authors are convinced that the result holds a general scope. Especially, it is expected that other techniques would approximately yield the same curves. Each curve as it is can be used as a look-up table to know whether the flow is unsteady. A consolidation of these results would have to be carried out to take into account additional parameters that enter, in principle, the threshold function, such as the turbulent level of the wind tunnel and the surface quality of the model, to name a few.

This curve is shown in Fig. 11. In the laminar case, the support of the curve is the entire range of $\alpha$ considered in the analysis while in the turbulent case, the support is limited to $\alpha>3^{\circ}$ (this limit is indicated by a double line sign). Points $\left(\mathrm{M}_{0}, \alpha\right)$ above each curve exhibit an unsteady periodic flow field while those below do not. The limited support of the

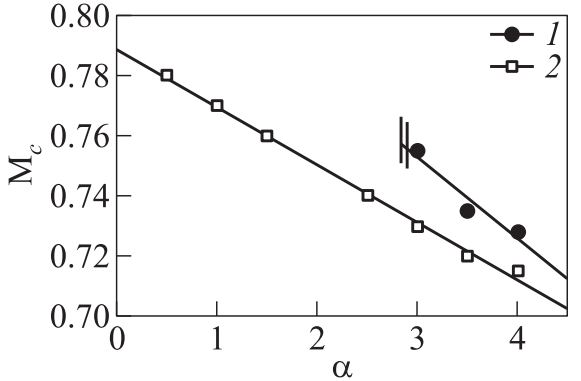

Figure 11 Threshold curves of buffet in transition forced at $7 \%$ (turbulent) (1) and free cases (2) as a function of $\alpha$. In the turbulent case, buffet occurs only for $\alpha>3^{\circ}$. The absence of buffet below that angle is shown by the double line mark turbulent curve is not related to an absence of shock, since shock appears below $\alpha=3^{\circ}$. It is observed that instead of oscillating, the flow behind the shock only detaches, without any unsteadiness.

\section{DISCUSSION}

\subsection{Acoustic Feedback Mechanism}

An acoustic feedback loop scenario has been proposed by several authors (see, for example, [2-4]) to explain the turbulent buffet. The physics embedded in this 
(a)

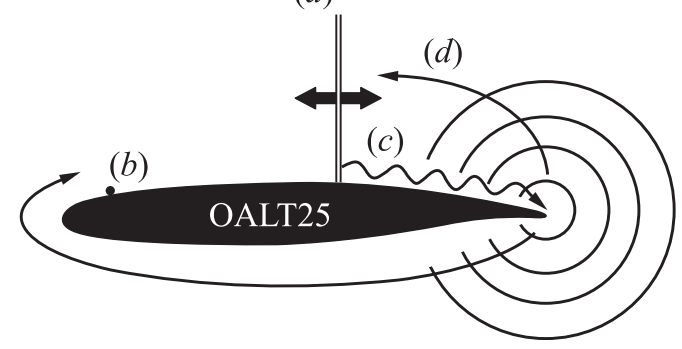

Figure 12 Schematic of the acoustic feedback loop scenario: (a) - shock oscillations; $(b)$ - tripping device; $(c)$ - hydrodynamic waves; and $(d)$ acoustic feedback theory is schematized in Fig. 12 and is to be understood as follows: the shock motion induces hydrodynamic waves (c) traveling downstream. An acoustic wave $(d)$ is formed when these waves hit the trailing edge. The acoustic wave hits the shock back disturbing the shock again and closing the loop of the interaction. Note that due to the tripping in $(b)$, the boundary layer at the shock in (a) is turbulent.

The scaling of this loop relies on the convection time $\tau_{c}$ of the hydrodynamic waves from the shock to the trailing edge and on the propagation time $\tau_{a}$ of the acoustic wave from the trailing edge back to the shock. The total time $\tau=\tau_{c}+\tau_{a}$ allows one to form a frequency characteristic of the loop. The acoustic wave may hit the shock by traveling along the upper surface or along the lower surface. However, the upper path is physically more consistent than the lower path because it reaches directly the shock while the lower path implies strong curvature at the leading edge which will likely decrease the wave strength.

The scenario is applied to the turbulent buffet case $\left(\mathrm{M}_{0}, \alpha\right)=\left(0.735,4^{\circ}\right)$ discussed previously. The convection time $\tau_{c}$ of the hydrodynamic waves is measured using the cross correlation between the pressure signals on the upper surface. An illustration of the convection speed is given in Fig. 13a which gives a view on the propagation of the information from the shock toward the trailing edge using a lateral stack of the pressure signals available at the upper surface. It can be noticed from this figure that the wave propagates at constant speed. The speed is measured to be $U_{c}=18 \mathrm{~m} / \mathrm{s}=0.072 U_{0}$, in agreement with $[1,3]$. The associated time scale is $\tau_{c}=l_{c} U_{c}$ where $l_{c} \approx 0.5 c$ is the distance from the shock to the trailing edge (the shock is located at $50 \%$ of chord, see Fig. $5 b$ ), yielding $\tau_{c} \simeq 6.4 \mathrm{~ms}$. The acoustic waves travel at a speed approximately equal to $U_{a} \simeq a_{0}-U_{0}=0.36 U_{0}$ leading to a characteristic time scale $\tau_{a}=l_{a} / U_{a}$ with $l_{a}=0.5 \mathrm{c}$ and, finally, $\tau_{a}=1.3 \mathrm{~ms}$. The frequency associated with the feedback loop is then given by $f=\left(\tau_{c}+\tau_{a}\right)^{-1}$ and is about $110 \mathrm{~Hz}$. This is in reasonable agreement with the experimental value $(75 \mathrm{~Hz})$, although slightly too high. Such an overestimation was also noticed by Jacquin et al. [3].

The application of the acoustic feedback loop scenario to the laminar case is now considered. Figure $13 b$ shows the propagation of the information over the pressure signals set side by side and spatially interpolated. Unlike the turbulent case, information does not propagate at constant speed. An acceleration occurs 


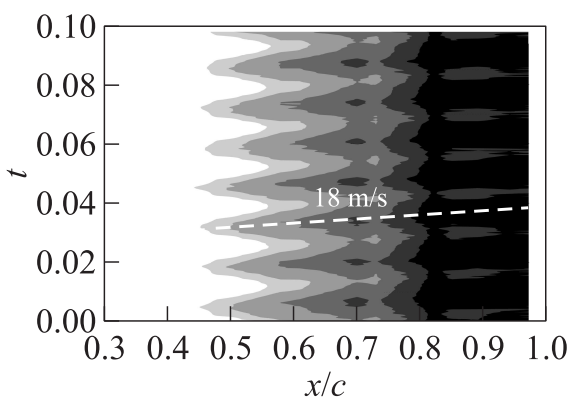

(a)

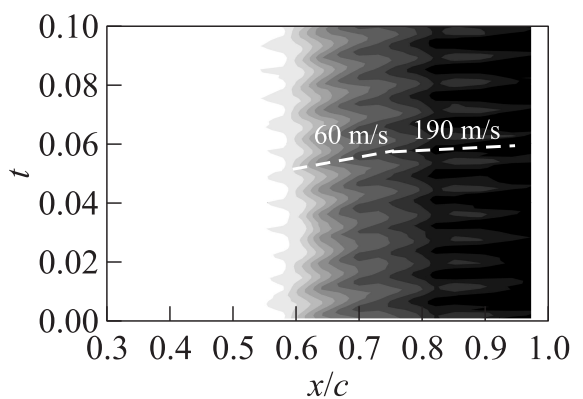

(b)

Figure 13 Value of the pressure as a function of time and space for $\left(\mathrm{M}_{0}, \alpha\right)$ $=\left(0.735,4^{\circ}\right)$ : (a) turbulent case with tripping at $7 \%$ of chord; and (b) laminar case. The figure is obtained by stacking the pressure signals of the pressure transducers side by side and by linearly interpolating them spatially. Only the upper surface of the wing is shown

at about $75 \%$ of chord, changing from 60 to $190 \mathrm{~m} / \mathrm{s}$. The total travel time is thus $\tau_{c}=\tau_{c 1}+\tau_{c 2}$ and $\tau_{c 1 / 2}=l_{c 1 / 2} / U_{c 1 / 2}$ with $l_{c 1}=0.15 c$, the distance from the shock to the change of propagation speed, and $l_{c 2}=0.25 c$, the remaining distance to the trailing edge. The value $\tau_{c}=0.9 \mathrm{~ms}$ is obtained. From the turbulent analysis, it can be inferred that the time for the acoustic feedback is $\tau_{a}=1 \mathrm{~ms}$ given that now $l_{a}=0.4 c$ while $U_{a}$ is unchanged. The loop frequency yields $f$ $\simeq 530 \mathrm{~Hz}$ which is much lower than the shock frequency observed experimentally. Thus, in the laminar case, the acoustic feedback scenario does not work well.

\subsection{Vortex Shedding and Separation Bubble Breathing}

Two other scenarios are presented instead to discuss the possible sources of unsteadiness. The first one relies on the observation that the Strouhal number of laminar buffet based on airfoil thickness is $S_{t}=0.19$ for $\mathrm{M}_{0}=0.735$. Such a value of Strouhal number is reminiscent of classical vortex shedding, implying that laminar buffet could result from a von Kármán type of flow instability. In fact, the flapping of the separated flow behind the shock, which generates the large vertical black spread behind the shock in Fig. 8b, coincides well with this analysis. Moreover, the existence of such an unstable mode was found by Bouhadji and Braza [13] for the flow past an aerofoil at nil incidence. Their analysis tackled the global stability of the flow at a fixed Reynolds number (Re $=10000)$, purely laminar case, and various Mach numbers up to the transonic regime. The unstable mode labeled $\mathrm{I}$ in their analysis exhibits a chord-based 
Strouhal number between 1.4 and 1.8 depending on the Mach number, which is of the same order of magnitude as the present value and corresponds to the vortex shedding behind the airfoil.

The second scenario is based on the model of the breathing of the separation bubble recently proposed by Piponniau et al. [14] to explain the low-frequency shock unsteadiness observed quite systematically in shock-boundary layer interactions with mean separation. While their analysis was applied to a turbulent flow configuration, the main ingredients, i. e., mean separation and the associated generation of vortices in the mixing layer separating the bubble and the external flow, are also present in the laminar case. The existence of the laminar separation was evidenced by the Schlieren images in Fig. 7 and by the numerical simulation results in Fig. 4b. The breathing of the bubble corresponds to the periodic ejection of fluid from the recirculation and the necessary fluid entrainment required to reform the bubble so as to make it present on the mean. The frequency scaling of this breathing is described by a simple model of these two phenomena and relies on the diffusion rate of the mixing layer $\delta_{w}^{\prime}$, the convective Mach number $\mathrm{M}_{c}$, and the ratios $r=u_{2} / u_{1}$ and $s=\rho_{2} / \rho_{1}$ that characterize it. In the present case, the intensity of the reverse flow is unknown experimentally but the RANS simulations as the one shown in Fig. 4 allows to give an estimate. Doing so in the buffet case yields $r \simeq-0.1, s \simeq 0.7$ and $\mathrm{M}_{c} \simeq 0.5$. With this, the scaling functions provided by Piponniau et al. [14] yield $g(r, s) \simeq 0.016$ and $\Phi\left(\mathrm{M}_{c}\right) \simeq 0.6$ and, as a result, the Strouhal number based on the length $l$ of the bubble and the external velocity $u_{1}$ is equal $S_{l \text {,theo }}=\Phi\left(\mathrm{M}_{c}\right) g(r, s) l / h \simeq 0.7$ where $l / h \simeq 75$. This should be compared to the experimental value $S_{l}=f l / u_{1}=0.1$, showing poor agreement. This suggests that the breathing of the bubble, were it to occur, may not be able to cause the observed unsteadiness, as it yields a much higher frequency. A reason for this high-frequency signature is the very large aspect ratio of the bubble, which tends to accelerate the rate of flow ingestion in the bubble and the consecutive ejection of fluid. However, owing to the large uncertainties present in the various quantities that had to be evaluated, the results should not be taken as conclusive at this stage.

\subsection{Effect of Delayed Tripping at $40 \%$ of Chord}

The effect of setting the boundary layer turbulent at $40 \%$ of chord is now analyzed. This scenario may be of interest to the laminar situation when an early transition occurs, for instance, in case a roughness gets stuck on the surface. In this situation, it is useful to know what the shock dynamics becomes and, especially, it must be ensured that nothing critical arises. As a primary evaluation of this, the fact that the forced transition at $7 \%$ case becomes unsteady at a higher threshold compared to the laminar setup was the first piece of information that nothing worst should occur. Testing the case at $40 \%$ will also help evaluate the 


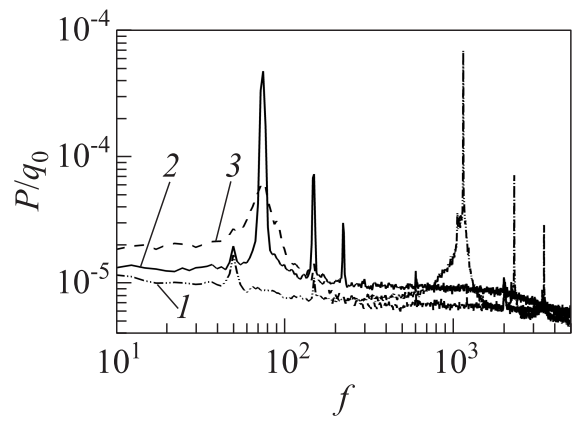

(a)

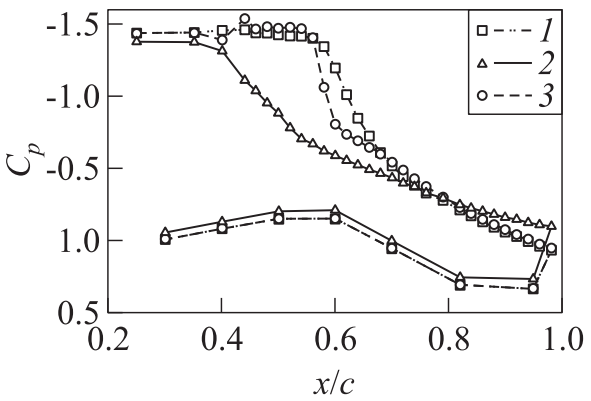

(b)

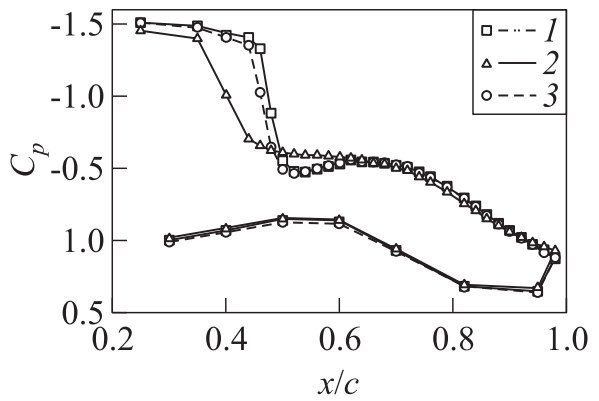

(c)

Figure 14 Comparison between the transition free (1), 7\% (2), and 40\% (3) cases: (a) pressure spectra in the unsteady regime; and mean $C_{p}$ distributions for $\left(\mathrm{M}_{0}, \alpha\right)$ $=\left(0.735,4^{\circ}\right)(b)$ and $\left(0.705,3.5^{\circ}\right)(c)$

sensibility of the previous findings upon the location of the laminar/turbulent transition.

The case of transition at $40 \%$ of chord is thus compared to the other tripping cases. Figure 14 shows the pressure spectra and $C_{p}$ distributions for the three test cases. Pressure spectra in the unsteady regime in Fig. $14 a$ show an interesting result, which is that unlike the first two tripping cases explored so far, the $40 \%$ case features a much attenuated and broader peak, the frequency of which matches the turbulent case at $7 \%$ tripping. In particular, no unsteadiness is observed close to laminar shock destabilization.

The $C_{p}$ distributions evaluate the influence of tripping the boundary layer before or after laminar separation. Thus, two cases are considered that feature laminar separation either after (Fig. 14b) or before (Fig. 14c) $40 \%$ of chord. Note that the location of laminar separation is deduced from a qualitative analysis of the $C_{p}$ distribution, which was illustrated earlier in Fig. $5 d$. In Fig. 14b, the 
compression of the flow upstream of the shock occurs after $40 \%$ of chord and the tripping is thus clearly upstream. In Fig. 14c, laminar separation occurs at about $40 \%$ and the tripping tape is placed at that location.

In case of tripping upstream of laminar separation, it turns out that laminar separation disappears. The $C_{p}$ distribution remains at the plateau level up to the shock, which also forms more upstream, following the trend of the fully turbulent case (tripping 7\%). On the contrary, tripping the boundary layer downstream of laminar separation, as is the case in Fig. $14 c$, seems to modify the flow only negligibly. Such results would need refinement to be fully conclusive but at this point, they suggest that the modification of the wing aerodynamics is only impacted if the boundary layer becomes turbulent ahead of laminar separation, while slightly disturbing the boundary layer inside the bubble has no effect.

Note in the end that the tripping acts as a stationary and small forcing upon the flow. In particular, the presented analysis does not encompass unsteady and large amplitude forcing which could lead to different conclusions.

\section{CONCLUDING REMARKS}

The shock dynamics over a laminar transonic airfoil has been investigated in a wind tunnel experiment by varying the Mach number, the angle of attack, and the state of the boundary layer interacting with the shock. The goals were to extend the current understanding on buffet to laminar flows and provide a database to validate the numerical simulations. The findings were obtained by various measurement tools, namely, pressure sensing, high-speed Schlieren imagery, and thermography. Complementary RANS simulations were carried out in the steady regime to validate the numerical model, including the compressible AHD transition criterion.

It has been shown that when the flow is laminar up to the shock and the couple $\left(\mathrm{M}_{0}, \alpha\right)$ is raised above a given threshold, a strong oscillation of the flow arises, in which the shock foot moves at a Strouhal number based on chord of 1 and the flow periodically detaches behind the shock down to the trailing edge. The phenomenon shares similarities with turbulent buffet but is much faster and with only little shock movement. Turbulent buffet is also found at a chord-based Strouhal number of about 0.07 in agreement with previous studies. The application of the acoustic feedback loop scenario, which works well in the turbulent case, shows poor agreement with the experimental observations in the laminar setup. Two additional scenarios have been presented, one that relies on the airfoil thickness based Strouhal number of 0.19 peculiar to the laminar interaction that suggests dynamics driven by vortex shedding and the second one which is based on the possible breathing dynamics of the laminar separation bubble present under the shock foot in the laminar case. Both of them 
clearly require further analysis. Other scenarios based on influences coming from perturbations present in the upstream boundary layer, thus as the one proposed by Beresh et al. [15], have not been evaluated in the present analysis.

The laminar and turbulent, artificially tripped case at $7 \%$ of chord, thresholds in the $\left(\mathrm{M}_{0}, \alpha\right)$ plane have been derived from the experimental data. The results show that laminar buffet sets in prior to turbulent buffet with a margin of about $\Delta \alpha \simeq 0.5^{\circ}$ and $\Delta \mathrm{M}_{0} \simeq 0.01$ in the range of parameters investigated. Moreover, the case of tripping at $40 \%$ of chord was analyzed showing that from an initial laminar case, the shock dynamics is strongly impacted by tripping the boundary layer right before laminar separation, inducing a disappearance of the unsteadiness at the high-frequency laminar peak and the occurrence of an attenuated dynamics centered about the low-frequency turbulent peak. Further analysis of the phenomenon is clearly needed to complete the current set of results.

\section{ACKNOWLEDGMENTS}

The authors would like to thank the reviewers for their fruitful comments on the present work. The paper greatly benefited from their inputs. This work has been supported by the European FP7 Project BUTERFLI (FP7-AAT-2013.8-1RTDRUSSIA) program.

\section{REFERENCES}

1. McDevitt, J. B., and A.F. Okuno. 1985. Static and dynamic pressure measurements on a NACA 0012 airfoil in the Ames high Reynolds number facility. National Aeronautics and Space Administration, Scientific and Technical Information Branch. Vol. 2485. 78 p.

2. Lee, B. H. K., and F. C. Tang. 1989. Transonic buffet of a supercritical airfoil with trailing-edge flap. J. Aircraft 26(5):459-464.

3. Jacquin, L., P. Molton, S. Deck, B. Maury, and D. Soulevant. 2009. Experimental study of shock oscillation over a transonic supercritical profile. AIAA J. 47(9):19851994.

4. Crouch, J. D., A. Garbaruk, D. Magidov, and A. Travin. 2009. Origin of transonic buffet on aerofoils. J. Fluid Mech. 628:357-369.

5. Dor, J. B., A. Mignosi, A. Seraudie, and B. Benoit. 1989. Wind tunnel studies of natural shock wave separation instabilities for transonic airfoil tests. Symposium Transsonicum III. Springer. 417-427.

6. Bartels, R. E. 1997. Computation of shock buffet onset for conventional and supercritical airfoil. AIAA Paper No.97-0833.

7. Deck, S. 2005. Numerical simulation of transonic buffet over a supercritical airfoil. AIAA J. 43(7):1556-1566. 
8. Xiao, Q., H. Tsai, and F. Liu. 2006. Numerical study of transonic buffet on a supercritical airfoil. AIAA J. 44(3):620-628.

9. Schlichting, H., and K. Gersten. 2000. Boundary-layer theory. Berlin-Heidelberg: Springer. $834 \mathrm{p}$.

10. Spalart, P. R., and S. R. Allmaras. 1992. A one-equation turbulence model for aerodynamic flows. 30th AIAA Aerospace Sciences Meeting and Exhibit.

11. Arnal, D. 1989. Transition prediction in transonic flow. Symposium Transsonicum III. Springer. 253-262.

12. Gleyzes, C., J. Cousteix, and J. L. Bonnet. 1985. Theoretical and experimental study of low Reynolds number transitional separation bubbles. Conference on Low Reynolds Number Airfoil Aerodynamics. Notre Dame, IN. 137-152.

13. Bouhadji, A., and M. Braza. 2003. Organised modes and shock-vortex interaction in unsteady viscous transonic flows around an aerofoil. Part I: Mach number effect. Comput. Fluid. 32(9):1233-1260.

14. Piponniau, S., J. P. Dussauge, J. F. Debieve, and P. Dupont. 2009. A simple model for low-frequency unsteadiness in shock-induced separation. J. Fluid Mech. 629:87108.

15. Beresh, S. J., N. T. Clemens, and D. S. Dolling. 2002. Relationship between upstream turbulent boundary-layer velocity fluctuations and separation shock unsteadiness. AIAA J. 40(12):2412-2422. 